\title{
Absence of electroencephalographic seizure activity in patients treated for head injury with an intracranial pressure-targeted therapy
}

\author{
Clinical article
}

\author{
Magnus Olivecrona, M.D., ${ }^{1}$ Bo Zetterlund, M.D., ${ }^{2}$ Marie Rodling-Wahlström, M.D., ${ }^{3}$ \\ Silvana Naredi, M.D., Ph.D., ${ }^{3}$ and Lars-Owe D. Koskinen, M.D., Ph.D. ${ }^{1}$
}

Departments of ${ }^{1}$ Neurosurgery, ${ }^{2}$ Clinical Neurophysiology, and ${ }^{3}$ Anaesthesiology, University Hospital, Umeå, Sweden

\begin{abstract}
Object. The authors prospectively studied the occurrence of clinical and nonclinical electroencephalographically verified seizures during treatment with an intracranial pressure (ICP)-targeted protocol in patients with traumatic brain injury (TBI).

Methods. All patients treated for TBI at the Department of Neurosurgery, University Hospital Umeå, Sweden, were eligible for the study. The inclusion was consecutive and based on the availability of the electroencephalographic (EEG) monitoring equipment. Patients were included irrespective of pupil size, pupil reaction, or level of consciousness as long as their first measured cerebral perfusion pressure was $>10 \mathrm{~mm} \mathrm{Hg}$. The patients were treated in a protocol-guided manner with an ICP-targeted treatment based on the Lund concept. The patients were continuously sedated with midazolam, fentanyl, propofol, or thiopental, or combinations thereof. Five-lead continuous EEG monitoring was performed with the electrodes at F3, F4, P3, P4, and a midline reference. Sensitivity was set at 100 $\mu \mathrm{V}$ per $\mathrm{cm}$ and filter settings $0.5-70 \mathrm{~Hz}$. Amplitude-integrated EEG recording and relative band power trends were displayed. The trends were analyzed offline by trained clinical neurophysiologists.

Results. Forty-seven patients (mean age 40 years) were studied. Their median Glasgow Coma Scale score at the time of sedation and intubation was 6 (range 3-15). In $8.5 \%$ of the patients clinical seizures were observed before sedation and intubation. Continuous EEG monitoring was performed for a total of 7334 hours. During this time neither EEG nor clinical seizures were observed.

Conclusions. Our protocol-guided ICP targeted treatment seems to protect patients with severe TBI from clinical and subclinical seizures and thus reduces the risk of secondary brain injury. (DOI: 10.3171/2008.4.17538)
\end{abstract}

\section{KEY WoRdS • continuous EEG • head injury • seizure}

$\mathrm{D}$ URING the last decades, the importance of the use of cEEG monitoring in the setting of cerebral intensive care has been recognized. $3,4,11,13,16,18,24-32,33-37,39$ The rational for cEEG monitoring is to detect events that can result in secondary brain injury or to detect signs of secondary brain injury. Secondary events detectable by electroencephalography are ischemia and seizures. High frequencies of seizures $(8-55 \%)$ have been reported in neurointensive care settings irrespective of the underlying disease treated. ${ }^{5,6,10,27,32,37}$ The majority of these seizures are said to be nonconvulsive, and thus clinically

\footnotetext{
Abbreviations used in this paper: $\mathrm{AED}=$ antiepileptic drug; $\mathrm{BP}=$ blood pressure; $\mathrm{cEEG}=$ continuous electroencephalographic; GCS = Glasgow Coma Scale; ICP = intracranial pressure; ICU = intensive care unit; $\mathrm{SE}=$ status epilepticus; $\mathrm{TBI}=$ traumatic brain injury.
}

not detectable. It has further been claimed that patients who suffer seizures during intensive care have a poorer prognosis than those without seizures., 3,12,30,39

At the Department of Neurosurgery at Umeå University Hospital we have treated patients with severe TBI in a protocol-driven manner since 1994 . We have used an ICP-targeted therapy based on the Lund concept. ${ }^{1,7-9}$ In 2003 we introduced continuous encephalography to our standard monitoring to follow the sedation level, especially during the time thiopental is used for sedation.

With the introduction of cEEG monitoring, we decided to investigate the frequency of nonconvulsive and convulsive seizures. The present study is a prospective study of patients with TBI who were treated at our department and in whom cEEG monitoring was used. Our specific aim in undertaking this study was to describe the occurrence of EEG seizures in our patients treated for TBI according to our ICP-targeted protocol. We hypoth- 
esized that our treatment protocol, which includes continuous sedation, would result in a low rate of seizures.

\section{Methods}

\section{Patient Population}

All referring hospitals in the catchment area of our department refer patients with TBI to us. Patients younger than 80 years of age with TBI, irrespective of neurological condition, complicating illness, or other injury, were accepted for admittance during the study period.

All patients treated for TBI at our department between March 1, 2004, and September 30, 2006, were eligible for the study. Inclusion was consecutive and based on the availability of the EEG monitoring equipment. There was no systematic exclusion of patients. For acceptance into the study a patient had to have suffered a verified head injury and be in need of cerebral intensive care as a result of the trauma. Patients were included irrespective of pupil size, pupil reactivity, or level of consciousness as long as their first measured cerebral perfusion pressure was $\geq 10$ $\mathrm{mm} \mathrm{Hg}$. The patients had to have arrived in our hospital $\leq 24$ hours after the trauma.

\section{Monitoring}

Intracranial pressure was monitored in all patients using either a Codman MicroSensor (Johnson \& Johnson Professional, Inc.) or by means of ventriculostomy. The MicroSensor was calibrated according to the manufacturer's instructions. The preauricular level was used as zero-level for the ventriculostomy. All patients were initially treated in the supine position with no head elevation. Arterial BP was continuously monitored using the radial artery, with heart level used as zero-level. Mean arterial BP and other physiological parameters such as systolic and diastolic BP, oxygen saturation, heart rate, and respiratory parameters were continuously monitored, and the resulting data were stored digitally using the Marquette Solar system (GE Healthcare). Data pertaining to drugs and fluids administered to the patients were stored in a computer system using the Picis system (Picis, Inc.).

\section{Treatment}

Our protocol-guided treatment largely followed the original guidelines for the Lund concept and has been thoroughly described and published elsewhere. ${ }^{22}$

All patients were continuously sedated to a comfortable level allowing them to cough, initially using midazolam and fentanyl. Expansive mass lesions were aggressively treated surgically. The patients were all ventilated in a controlled breathing mode, with the aim of maintaining a normal $\mathrm{pH}$ and $\mathrm{a} \mathrm{PaCO}_{2}$ between $4.5-5.5 \mathrm{kPa}$ and a $\mathrm{PaO}_{2}$ above $12 \mathrm{kPa}$. Hyperventilation was not allowed.

No prophylactic AED treatment was administered to the patients after admittance in our department.

\section{Electroencephalography}

Two NicoletOne Monitors (VIASYS Healthcare, Inc.) were used. Five needle electrodes were placed sub- cutaneously by trained neurophysiological technicians at F3, F4, P3, P4, and a midline reference. The electrode positions sometimes had to be adjusted due to the surgical wounds. The raw F3-P3 and F4-P4 EEG tracings were displayed continuously at the bedside. Sensitivity was set at $100 \mu \mathrm{V}$ per $\mathrm{cm}$ and filter settings at $0.5-70 \mathrm{~Hz}$. Amplitude-integrated EEG data and relative band power trends were displayed at a resolution of 2 hours per screen. In our experience, these trends are sensitive to most types of epileptic seizure activity. The continuous display at the bedside would allow the nurse and physician caring for the patients to detect a seizure immediately. At least twice a day the trends were analyzed offline by 1 of 3 senior clinical neurophysiologists. Samples of raw EEG data were reviewed, often even in reference derivations and with higher sensitivity $(50 \mu \mathrm{V} / \mathrm{cm})$. Interictal epileptiform activity and nonspecific slowing were not looked for systematically.

\section{Statistics}

Results are reported as means \pm standard errors of the means or in cases of nonparametric variables as medians and ranges.

\section{Ethical Approval}

This study was approved by the local ethics committee (2000-00-175).

\section{Results}

Forty-seven patients (33 men and 14 women) were included in the study. The mean age at trauma was $40.0 \pm 2.5$ years (range 11-79 years). The median GCS score at intubation and sedation was 6 (range 3-15) (see Fig. 1). Four patients were intubated with GCS scores $>8: 2$ had complex facial fractures, 1 had severe motor restlessness, and 1 was intubated before transportation to our hospital due to hematemesis. Pathological changes (Fig. 2) as classified according to Marshall and colleagues ${ }^{19}$ were evident on initial CT in all but 2 of the 47 patients. Subsequent CT did not reveal pathological changes in either of these patients, although the ICP increased to $>20 \mathrm{~mm} \mathrm{Hg}$. One of the 47 patients had a history of juvenile myoclonic epilepsy, treated with valproate. At the time the TBI was sustained this patient had been seizure free for $>1$ year.

During the study period, 7334 hours ( 306 days) of EEG data were recorded. The mean duration of monitoring per patient was $156 \pm 11$ hours. Figure 3 shows a Kaplan-Meier plot of the duration of cEEG monitoring. The mean time between admission and initiation of monitoring was $51.1 \pm 5$ hours (median 35 hours, range 10-168 hours). The total ICU time was 13,676 hours; thus cEEG activity was monitored during 54\% of the total ICU time. During the whole study period no EEG seizures were found. In 4 patients $(8.5 \%)$, clinical seizures were observed before the start of monitoring; the seizures all occurred outside the hospital and before sedation and intubation. After sedation and intubation no clinical seizures were observed.

All the patients were continuously sedated with mi- 


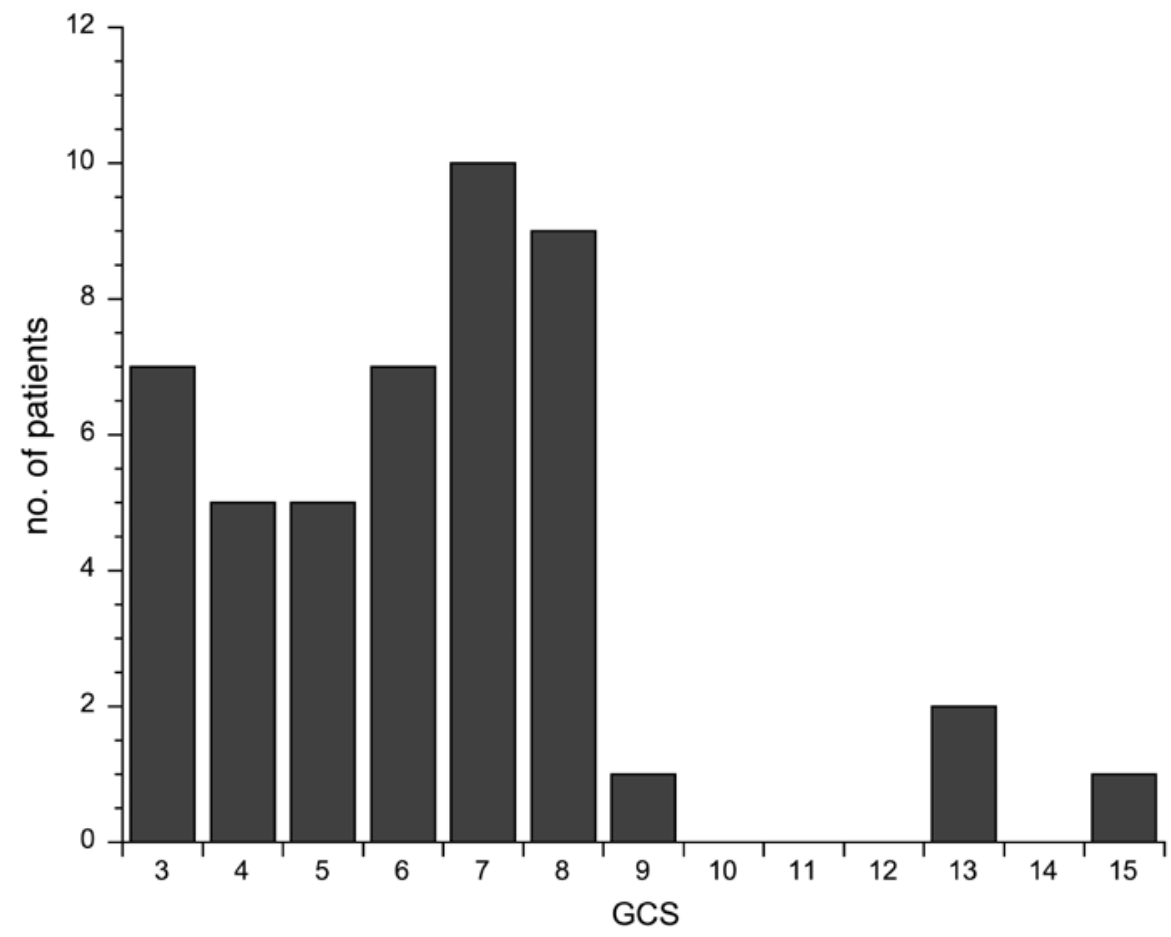

FIG. 1. Bar graph showing GCS scores at intubation and sedation in 47 patients. Values on the $y$ axis represent numbers of patients. dazolam, propofol, or thiopental, or combinations of the 3 sedative agents. During the period of cEEG monitoring the patients also received fentanyl. Metoprolol and clonidine were administered to reduce the sympathetic nervous system tone related to stress. The drugs were also administered to normalize the arterial BP. Figure 4 depicts the sedative drugs administered during the cEEG monitoring period. Only 8 patients had cEEG monitoring time without sedation, and then only at the end of the monitoring period (in 5 patients, the last day; in 2 patients, the 2 last days; and in 1 patient the last 5 of 16 days). The drugs and doses $(\mathrm{mg} / \mathrm{kg} /$ day) delivered to the patients during the monitoring period are shown in Fig. 5.

\section{Discussion}

Immediately after the accident and before sedation and intubation, clinical seizures were observed in $8.5 \%$ of the patients. We did not, however, find any EEG or clinical seizures in the included 47 patients with TBI during 7334 hours of EEG monitoring. This finding stands in contrast to the general conception that nonclinical seizures or SE is common in patients who require neurological intensive care for TBI.

All the patients in our study group were continuously sedated, according to our treatment protocol, with intravenously administered midazolam, propofol, thiopental, or a combination thereof. All these drugs have an antiepileptic effect and are used in the treatment of SE. The continuous use of these drugs could explain the absence of EEG seizures. There is no evidence that the use of conventional AEDs, such as valproate or phenytoin for early seizure prophylaxis in TBI would improve outcome. There are studies showing that the use of AEDs to pre- vent late posttraumatic seizures may have a negative influence on outcome. Therefore we do not use prophylactic AEDs. ${ }^{2}$

In addition to monitoring ICP, we monitor the neurological condition of our sedated patients by relying on clinical signs such as pupillary reaction and response to painful stimuli in combination with frequent CT scans of the brain. We have previously shown that monitoring ICP using the Codman MicroSensor is reliable and stable over time..$^{15}$ The cEEG monitoring in our department was introduced to monitor the level of sedation, especially when using thiopental, aiming at a continuous delta pattern. This gave us the opportunity to also study the occurrence of EEG seizures.

Our treatment protocol does not include an awakening test. The awakening test is a stressful procedure and can provoke seizures. We have observed that in patients with unstable ICP and a tendency toward higher ICP values, a reduction of sedation as in an awakening test tends to result in an elevated ICP. The cessation of sedation necessary for the awakening test may also contribute to seizures. With our established treatment of patients with TBI, we further lower the general stress mediated by the sympathetic nervous system by administering clonidine and metoprolol. ${ }^{23}$ The above-mentioned measures may separately or in combination explain or may have contributed to the absence of seizures in our study.

There have been several reports of a high frequency of EEG seizures in patients subjected to neurointensive care for various reasons. We have, however, found only 2 papers specifically reporting on EEG seizures in patients treated for TBI. Vespa and colleagues ${ }^{37}$ reported clinical and EEG seizures in $21(22 \%)$ of 94 patients who had suffered moderate to severe TBI. Sixteen (17\%) of 


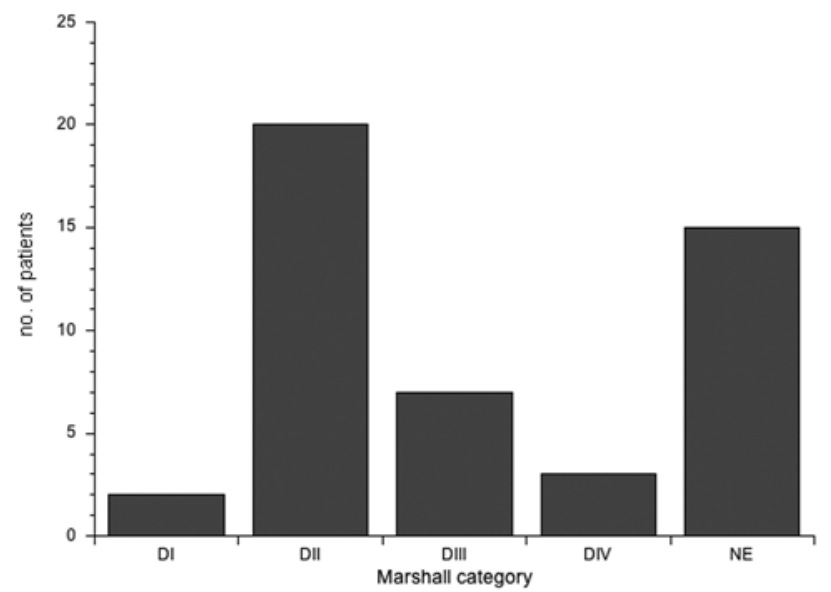

FIG. 2. Bar graph showing severity of TBI in 47 patients as demonstrated by the initial CT scans, assessed using the Marshall classification system. DI, DII, DIII, and DIV represent the Diffuse Injury Categories I-IV, respectively; NE = nonevacuated mass.

these patients experienced seizures in the hospital during cEEG monitoring and treatment. Five $(5 \%)$ had generalized tonic-clonic seizures before the initiation of cEEG monitoring. The median day of seizure occurrence in the monitored patients was the first day after the day of the injury. The patients in this study are said to have been managed in a protocol-guided fashion. The authors do not state anything about the eventual sedation of the patients or whether awakening tests were performed. All of these patients did receive prophylactic AED treatment. The mean cEEG monitoring time was $7.5 \pm 4$ days, and the monitoring started at a mean of $9.6 \pm 5.4$ hours after injury. In 2006, Ronne-Engström and Winkler ${ }^{27}$ reported on 70 patients with TBI. The patients were treated in a protocol-guided manner, using mild hyperventilation, ICP monitoring, surgical intervention, drainage of cerebrospinal fluid, and pharmacological control of BP. The authors stated that sedation with propofol and ketobemidone was used, but not whether this applied to all the patients. No prophylactic AED treatment was administered. They found EEG seizures in 20 patients (28\%). In another 3 patients (4\%) there were clinical overt seizures, but in these there was no EEG monitoring because of technical reasons. The median cEEG recording time in the patients was 58 hours (range 24-377 hours). The time between accident and seizure was reported to be $74 \pm 47$ hours. The median time between accident and arrival in our hospital is $\sim 8$ hours..$^{21,38}$ This is due to the size of our catchment area, $1000 \mathrm{~km}$ to the north, $600 \mathrm{~km}$ to the south, and 400 $\mathrm{km}$ to the west. Therefore, it is impossible for us to initiate EEG monitoring as early as Vespa et al. ${ }^{37}$ reported, but we are able to start well within the time frame reported by Ronne-Engström and Winkler.

It has been stated that the observed clinical or nonclinical seizures or nonconvulsive SE are seen early in the course of treatment of TBI or in the immediate posttrauma period. . $^{3,14,17,27,32,37}$ We started our monitoring $\sim 50$ hours after patients were admitted to our ICU. The later

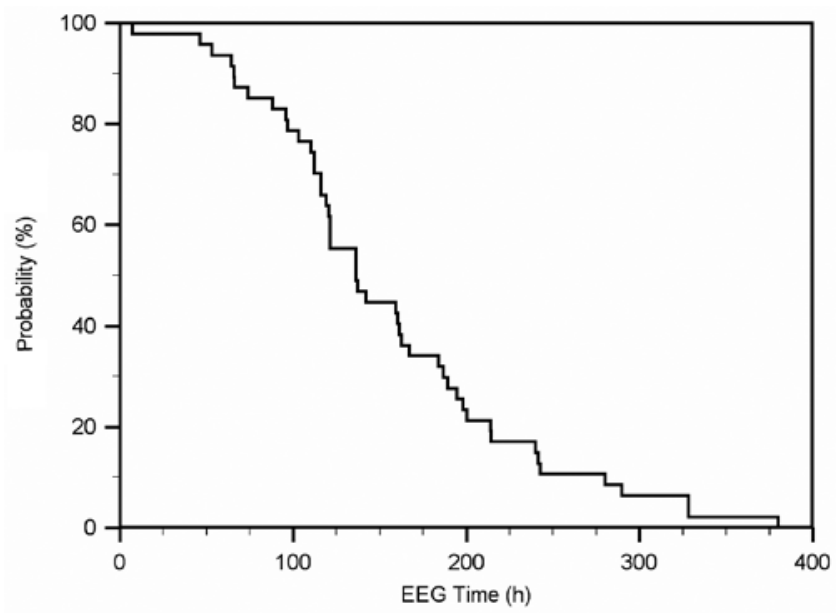

FIG. 3. Kaplan-Meier plot showing the probability of continued EEG monitoring over time.

start of EEG monitoring could be a partial explanation for the absence of EEG seizures. Nevertheless, we found this explanation not to be very plausible in the light of the long total monitoring time and the presumed high incidence of subclinical seizures. Furthermore, when we excluded the 4 cases in which the time between injury and initiation of cEEG monitoring exceeded 100 hours, the mean time to registration was $42.5 \pm 3.6$ hours (median 34 hours).

Most published studies of the occurrence of nonconvulsive, subclinical seizures have used EEG montages with higher numbers of electrodes than we chose to use. There are studies from authors using 14-channel 12-electrode montage ${ }^{35,37}$ or 11 electrodes, ${ }^{27}$ while the authors of other studies do not specify the montage or number of channels..$^{32}$ We chose to use 5 subdermal electrodes. This small number of electrodes has been used by Labar et al. ${ }^{16}$ and Theilen et al. ${ }^{31}$ The use of fewer electrodes reduces the spatial resolution, but it facilitates patient care in the ICU setting. In patients with severe TBIs who are treated in the ICU, head wounds, fresh surgical scars, and

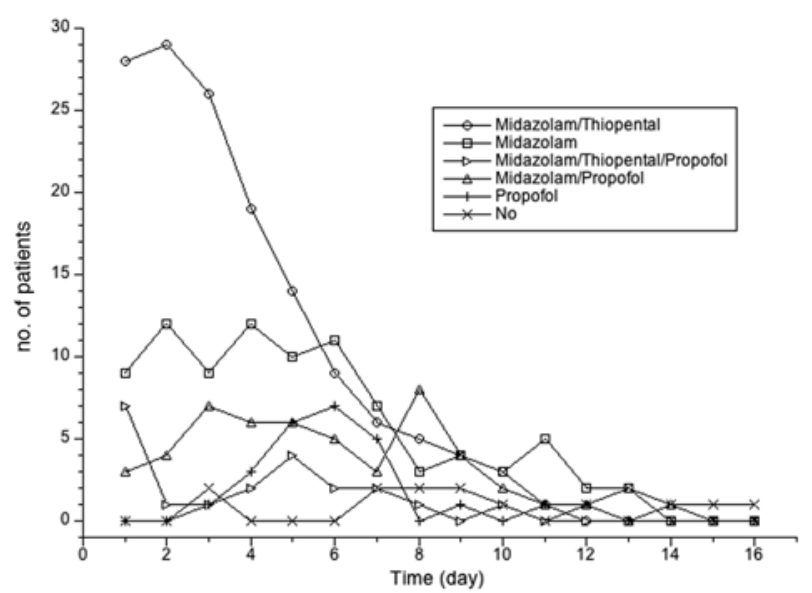

FIG. 4. Line graph showing the drugs delivered during cEEG monitoring. No = no sedative drug administered. Values on the $y$ axis represent numbers of patients. 


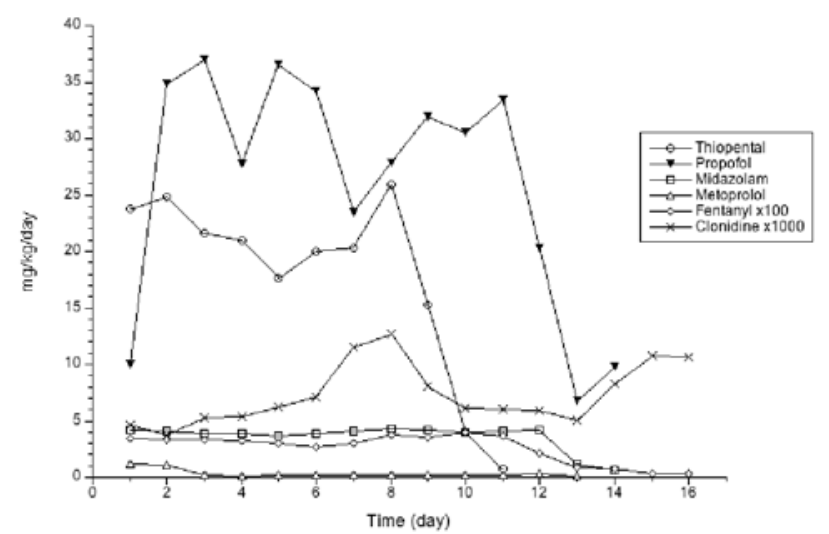

FIG. 5. Line graph showing the mean drug doses delivered per patient and day (mg/kg/day).

catheters may restrict the possibilities for electrode placement. Furthermore, the patients are frequently moved for examinations such as CT scans or transported to the operating room for further surgery; increased numbers of electrodes attached to a patient create more difficulties for the nursing staff in handling the patient. The absence of registered seizures could theoretically be a result of too few measuring points, although the findings of Labar et al. and Theilen et al. argue against this explanation. In a preliminary study of patients with nonconvulsive SE, Rosén found that it was possible to recognize $90 \%$ of the seizures in a 2-channel recording from F3, P3, F4, and P4 (I. Rosén, personal communication, 2007). Thus, we consider the use of 5 electrodes to be sufficient to detect EEG seizures in the clinical setting in the ICU.

It has been reported that patients suffering from clinical and subclinical seizures or SE during their treatment in neurointensive care have a poorer outcome in comparison with patients without seizures., ${ }^{3,39}$ Nevertheless, Towne and associates ${ }^{32}$ were unable to demonstrate a statistically significant difference in mortality between TBI patients with or without EEG seizures. It is known that all diseases affecting the brain can result in seizures and a brain under stress is prone to react with seizures. The seizures, whether clinical or not, are increasing the demands on the brain's metabolism, and therefore it is plausible that seizures in a head-injured patient can aggravate the patient's condition.

The fact that we did not detect any EEG seizures could be explained as a result of many factors. We have previously published our outcome results, ${ }^{20-22,38}$ which showed a favorable outcome in the range of $70 \%$ and a mortality of $<15 \%$. One factor contributing to the good outcome could be that we have a low frequency of or no EEG seizures. The reason for this could be found in the protocol-guided way in which the patients are treatedoptimizing the circulation; avoiding hypoxia, hypotension, and hypoperfusion of the brain; continuous sedation; aggressive surgery; and the avoidance of stressful events such as awakening tests. Moreover, we do not use hyperventilation, mannitol, or muscle relaxants, all known to provoke seizures in certain situations.

\section{Conclusions}

The goal of cerebral intensive care for TBI is to avoid secondary insults to the patient. The protocol-guided treatment we used for TBI seems to protect the patients from clinical and nonclinical seizures, and thus from 1 cause of secondary injuries. We would recommend the use of a treatment protocol that includes continuous sedation and ICP and EEG monitoring in the treatment of patients with severe TBI. Our use of electroencephalography not only provides information about the occurrence of seizures but also allows continuous monitoring of patients' sedation levels.

\section{Disclosure}

This study was supported by grants from the Department of Neurosciences at Umeå University, Umeå University Hospital General Foundation, Capio Research Foundation, and Tore Nilssons Foundation.

\section{Acknowledgments}

We thank the ICU personnel for their cooperation and support, including their acceptance of additional monitoring equipment, and the neurophysiology staff for their professional help with the technical details of the cEEG monitoring.

\section{References}

1. Asgeirsson B, Grande PO, Nordstrom $\mathrm{CH}$ : A new therapy of post-trauma brain oedema based on haemodynamic principles for brain volume regulation. Intensive Care Med 20: 260-267, 1994

2. Brain Trauma Foundation, American Association of Neurological Surgeons, Congress of Neurological Surgeons, Joint Section on Neurotrauma and Critical Care, AANS/CNS, Bratton SL, et al: Guidelines for the management of severe traumatic brain injury. XIII. Antiseizure prophylaxis. J Neurotrauma 24 (1 Suppl):S83-S86, 2007

3. Chiaretti A, De Benedictis R, Polidori G, Piastra M, Iannelli A, Di Rocco C: Early post-traumatic seizures in children with head injury. Childs Nerv Syst 16:862-866, 2000

4. Claassen J, Mayer SA: Continuous electroencephalographic monitoring in neurocritical care. Curr Neurol Neurosci Rep 2:534-540, 2002

5. Claassen J, Mayer SA, Kowalski RG, Emerson RG, Hirsch LJ: Detection of electrographic seizures with continuous EEG monitoring in critically ill patients. Neurology 62:1743-1748, 2004

6. Grand'Maison F, Reiher J, Leduc CP: Retrospective inventory of EEG abnormalities in partial status epilepticus. Electroencephalogr Clin Neurophysiol 79:264-270, 1991

7. Grände PO, Asgeirsson B, Nordström C: Aspects on the cerebral perfusion pressure during therapy of a traumatic head injury. Acta Anaesthesiol Scand Suppl 110:36-40, 1997

8. Grände PO, Asgeirsson B, Nordström CH: Physiologic principles for volume regulation of a tissue enclosed in a rigid shell with application to the injured brain. J Trauma 42 (2 Suppl):S23-S31, 1997

9. Grände PO, Asgeirsson B, Nordström CH: Volume-targeted therapy of increased intracranial pressure: the Lund concept unifies surgical and non-surgical treatments. Acta Anaesthesiol Scand 46:929-941, 2002

10. Jordan KG: Continuous EEG and evoked potential monitoring in the neuroscience intensive care unit. J Clin Neurophysiol 10:445-475, 1993

11. Jordan KG: Continuous EEG monitoring in the neuroscience 
intensive care unit and emergency department. J Clin Neurophysiol 16:14-39, 1999

12. Jordan KG: Neurophysiologic monitoring in the neuroscience intensive care unit. Neurol Clin 13:579-626, 1995

13. Jordan KG: Nonconvulsive status epilepticus in acute brain injury. J Clin Neurophysiol 16:332-353, 1999

14. Kollevold T: Immediate and early cerebral seizures after head injuries. Part I. J Oslo City Hosp 26:99-114, 1976

15. Koskinen LO, Olivecrona M: Clinical experience with the intraparenchymal intracranial pressure monitoring Codman MicroSensor system. Neurosurgery 56:693-698, 2005

16. Labar DR, Fisch BJ, Pedley TA, Fink ME, Solomon RA: Quantitative EEG monitoring for patients with subarachnoid hemorrhage. Electroencephalogr Clin Neurophysiol 78:325-332, 1991

17. Lee ST, Lui TN, Wong CW, Yeh YS, Tzaan WC: Early seizures after moderate closed head injury. Acta Neurochir (Wien) 137:151-154, 1995

18. Lowenstein DH, Aminoff MJ: Clinical and EEG features of status epilepticus in comatose patients. Neurology 42: 100-104, 1992

19. Marshall LF, Marshall SB, Klauber MR, Van Berkum Clark M, Eisenberg H M, Jane JA et al: A new classification of head injury based on computerized tomography. J Neurosurg 75: S14-S20, 1991

20. Naredi S, Edén E, Zäll S, Stephensen H, Rydenhag B: A standardized neurosurgical neurointensive therapy directed toward vasogenic edema after severe traumatic brain injury: clinical results. Intensive Care Med 24:446-451, 1998

21. Naredi S, Olivecrona M, Lindgren C, Ostlund AL, Grände PO, Koskinen LO: An outcome study of severe traumatic head injury using the "Lund therapy" with low-dose prostacyclin. Acta Anaesthesiol Scand 45:402-406, 2001

22. Olivecrona M, Rodling-Wahlström M, Naredi S, Koskinen LO: Effective ICP reduction by decompressive craniectomy in patients with severe traumatic brain injury treated by an ICP-targeted therapy. J Neurotrauma 24:927-935, 2007

23. Payen D, Quintin L, Plaisance P, Chiron B, Lhoste F: Head injury: clonidine decreases plasma catecholamines. Crit Care Med 18:392-395, 1990

24. Privitera M, Hoffman M, Moore JL, Jester D: EEG detection of nontonic-clonic status epilepticus in patients with altered consciousness. Epilepsy Res 18:155-166, 1994

25. Privitera MD, Strawsburg RH: Electroencephalographic monitoring in the emergency department. Emerg Med Clin North Am 12:1089-1100, 1994

26. Procaccio F, Polo A, Lanteri P, Sala F: Electrophysiologic monitoring in neurointensive care. Curr Opin Crit Care 7:74-80, 2001

27. Ronne-Engström E, Winkler T: Continuous EEG monitoring in patients with traumatic brain injury reveals a high incidence of epileptiform activity. Acta Neurol Scand 114:47-53, 2006
28. Scheuer ML: Continuous EEG monitoring in the intensive care unit. Epilepsia 43 (3 Suppl):114-127, 2002

29. Spencer EM, Bolsin SN: EEG monitoring in the intensive therapy unit. Intensive Care World 7:34-38, 1990

30. Synek VM: EEG abnormality grades and subdivisions of prognostic importance in traumatic and anoxic coma in adults. Clin Electroencephalogr 19:160-166, 1988

31. Theilen HJ, Ragaller M, Tschö U, May SA, Schackert G, Albrecht MD: Electroencephalogram silence ratio for early outcome prognosis in severe head trauma. Crit Care Med 28:3522-3529, 2000

32. Towne AR, Waterhouse EJ, Boggs JG, Garnett LK, Brown AJ, Smith JR Jr: Prevalence of nonconvulsive status epilepticus in comatose patients. Neurology 54:340-345, 2000

33. Vespa P: Continuous EEG monitoring for the detection of seizures in traumatic brain injury, infarction, and intracerebral hemorrhage: "to detect and protect". J Clin Neurophysiol 22:99-106, 2005

34. Vespa PM, Boscardin WJ, Hovda DA, McArthur DL, Nuwer MR, Martin NA, et al: Early and persistent impaired percent alpha variability on continuous electroencephalography monitoring as predictive of poor outcome after traumatic brain injury. J Neurosurg 97:84-92, 2002

35. Vespa PM, Nenov V, Nuwer MR: Continuous EEG monitoring in the intensive care unit: early findings and clinical efficacy. J Clin Neurophysiol 16:1-13, 1999

36. Vespa PM, Nuwer MR, Juhász C, Alexander M, Nenov V, Martin N, et al: Early detection of vasospasm after acute subarachnoid hemorrhage using continuous EEG ICU monitoring. Electroencephalogr Clin Neurophysiol 103:607-615, 1997

37. Vespa PM, Nuwer MR, Nenov V, Ronne-Engstrom E, Hovda DA, Bergsneider M, et al: Increased incidence and impact of nonconvulsive and convulsive seizures after traumatic brain injury as detected by continuous electroencephalographic monitoring. J Neurosurg 91:750-760, 1999

38. Wahlström MR, Olivecrona M, Koskinen LO, Rydenhag B, Naredi S: Severe traumatic brain injury in pediatric patients: treatment and outcome using an intracranial pressure targeted therapy-the Lund concept. Intensive Care Med 31:832-839, 2005

39. Young GB, Jordan KG, Doig GS: An assessment of nonconvulsive seizures in the intensive care unit using continuous EEG monitoring: an investigation of variables associated with mortality. Neurology 47:83-89, 1996

Manuscript submitted September 27, 2007.

Accepted April 23, 2008.

Please include this information when citing this paper: published online 08/29/2008; DOI: 10.3171.2008.4.17538.

Address correspondence to: Magnus Olivecrona, M.D., Department of Neurosurgery, University Hospital, SE 90185 Umeå, Sweden.email: magnus.olivecrona@vll.se. 